\title{
Extraordinary high-Q resonances in the scattering by a dielectric slab containing a grating of circular cylinders
}

\author{
V.O. Byelobrov ${ }^{1}$, T.M. Benson ${ }^{2}$ \\ ${ }^{1}$ Institute of Radio-Physics and Electronics NAS of Ukraine, Laboratory of Micro and Nano Optics, \\ 61085 Kharkiv, Ukraine \\ ${ }^{2}$ George Green Institute for Electromagnetics Research, University of Nottingham, \\ NG7 2KP, Nottingham, UK \\ E-mail:volodia.byelobrov@gmail.com
}

\begin{abstract}
An infinite dielectric wire grating in free space is known to possess very high$\mathrm{Q}$ resonances at the frequencies close to the Rayleigh anomalies. Still a practical implementation of such a resonator may require its embedding in a dielectric slab. In this paper, it is shown that this can bring additional improvement of the Q-factors. A dielectric slab with a symmetrically embedded dielectric-wire grating is investigated under the normal incidence of plane waves. Accurate numerical solutions are derived for the $E$ - and $H$-polarization cases. Associated eigenvalue problems are solved for the realvalued lasing frequencies and the threshold values of material gain.
\end{abstract}

Keywords: grating, sub-wavelength wires, reflection resonances, lasing thresholds.

Manuscript received 11.10.13; revised version received 15.01.14; accepted for publication 20.03.14; published online 31.03.14.

\section{Introduction}

A theoretical model of a grating of infinite dielectric cylinders placed periodically in free space and illuminated by a plane wave has been investigated as a fundamental electromagnetic problem since the 1950s see, for instance, [1-3]. The high-Q resonances that occur near the Rayleigh anomalies can be noticed, for instance, from Figs 2 and 3 of [3], however no attempt was done to explain them before analytical analysis was performed in [4]. Surprisingly, any investigation of the associated eigenvalue problems has been missing until very recently. Such a problem was studied in [5] in the lasing formulation, i.e. for the grating of active cylinders (quantum wires) able to behave as a gain medium under pumping. In this case, the eigenvalues are not the complex natural frequencies as in conventional (passivecavity) formulation, but the pairs of real numbers, the lasing frequencies and the associated values of the bulk material gain. Details of this model and its connection to the lasing can be found in [6]. For instance, it has been shown in that paper that the mode threshold is inverse proportional to both the mode Q-factor and the overlap coefficient between the gain medium (i.e. active region) and mode electric field.

In [5], we found that the quantum-wire grating possessed specific grating modes in addition to the more understandable natural modes of a single dielectric cylinder perturbed by the presence of other cylinders. The grating modes display counter-intuitive properties: (i) the threshold gets lower if the grating gets sparser, i.e. for the larger ratios of period to cylinder radius [5] and (ii) mode near-field is a characteristic wave standing along the grating and spreading over many periods in the normal to the grating direction [7]. The lasing frequencies correlate very well with the resonance frequencies in the plane-wave scattering by the same grating and in-resonance near fields resemble the natural 
fields of the grating modes [7]. Infinite extension of the structure, being idealisation of real scatterers, gives a good agreement with the scattering by a finite grating if the number of cylinders is 10 or larger, as it was shown for a grating of silver cylinders [8].

In practical applications, gratings are frequently embedded in dielectric slabs. A slab with embedded grating of circular cylinders (Fig. 1) obviously possesses the above mentioned properties plus can support the slab modes [9], now perturbed by the cylinders. Further we build an accurate model of such structure to reveal the impact of physical and geometrical parameters of the slab on the grating modes. A similar scattering structure was investigated in [10], however here we will concentrate not on the scattering properties of the composite structure but rather on the interaction between the slab modes and the grating modes. We will also emphasize the possibility of enhancement of the latter mode Q-factors by changing geometrical parameters of the slab. The chosen way to assess the mode quality in this paper is computation of the associated lasing threshold that is an eigenvalue of the corresponding homogeneous problem with cylinders made of a gain material. This is justified by the fact found in [6] that lasing threshold is inversely proportional to the mode Qfactor.

\section{Formulation and basic equations}

Consider a grating of the parallel to the $z$-axis and periodic along the $x$-axis circular cylinders (wires). The grating period is $p$, each cylinder radius is $a$ and their refractive index is $v$. This grating is embedded in a dielectric slab whose refractive index is $v_{s}$ and thickness is $d=d_{1}+d_{2}+2 a$, where $d_{1,2}$ are the distances between the grating and the upper/lower slab surface. Suppose that the electromagnetic field is time-harmonic $\sim \exp (-i \omega t)$ and does not vary along the $z$-axis, hence the field analysis problem is two-dimensional. Illumination by a plane wave incident from the upper half-space (in the negative direction of the $y$-axis) is investigated.

The function $U$, denoting the $H_{z}$ component of the electro-magnetic field in the $H$-polarization case and the $E_{z}$ in the $E$-polarization case, must satisfy the Helmholtz equation with appropriate wavenumber inside and outside of cylinders, the Sveshnikov radiation condition at infinity [11], the condition of local integrability of power, and the boundary conditions demanding continuity of the tangential field components at cylinder boundaries. The free space wavenumber is $k=\omega / c=$ $2 \pi / \lambda$, where $c$ is the free-space light velocity and $\lambda$ is the wavelength, while inside the cylinders it is $k v$ and inside the slab $k v_{s}$. Besides, in this paper we will also use the normalized frequency as the ratio of period to wavelength, $\sigma=p / \lambda=k v_{s} p / 2 \pi$.
The field inside the slab but outside the grating $(a<|y|)<a+d_{1,2}$ and in outer space $(|y|>d / 2)$ can be represented as Floquet series of the incoming harmonics $\left\{a_{l}^{i} e^{-i \tau \kappa^{i} y+i \pi_{l} k^{i} x}\right\}$ and the outgoing ones $\left\{b_{l}^{i} e^{i \tau_{l} \kappa^{i} y+i \pi_{l} k^{i} x}\right\}$, where $\pi_{l}=l / \sigma, \tau_{l}=i\left(\pi_{l}^{2}-1\right)^{1 / 2}$, and the index $i$ corresponds to the domain so that $\kappa^{1}=k$ in free space and $\kappa^{2}=k v_{s}$ in the slab. Briefly the field and the incident wave can be written as a vector,

$U^{i}=\left(\begin{array}{c}a^{i} \\ b^{i}\end{array}\right), \quad U^{\text {incident }}=\left(\begin{array}{c}a^{\text {incident }} \\ 0\end{array}\right)$.

The total scattering matrix $A$ that characterises a slab with a grating has a block-type nature and can be presented as

$\left(\begin{array}{l}t \\ 0\end{array}\right)=A\left(\begin{array}{c}a^{\text {incident }} \\ r\end{array}\right), \quad A=\left(\begin{array}{cc}A^{I I} & A^{I} \\ A^{I I I} & A^{I V}\end{array}\right)$.

Here $\quad r=\left\{r_{l} e^{i \tau_{l} \kappa y+i \pi_{l} k x}\right\}$ and $t=\left\{t_{l} e^{-i \tau_{l} \kappa y+i \pi_{l} k x}\right\}$ are the vectors of the reflected and transmitted Floquet harmonics, respectively. Then the reflectance and transmittance are expressed as

$R(\sigma)=\sum_{|i|<\sigma} \tau_{i}\left|r_{i}\right|^{2}, \quad T(\sigma)=\sum_{|i|<\sigma} \tau_{i}\left|\delta_{i}^{0}+t_{i}\right|^{2}$.

The matrix $A$ is built by successive multiplication of the matrices that characterize the transmission of the incident field (1) throughout each elementary scatterer,

$$
A=F^{1} S^{1} G S^{2} F^{2},
$$

where $F^{i}$ are the transfer matrices for the upper and lower interfaces of the slab. They can be expressed using well-known Fresnel matrices as

$$
F^{i}=\left(\begin{array}{cc}
T^{+}-R^{-}\left(T^{-}\right)^{-1} R^{+} & R^{-}\left(T^{-}\right)^{-1} \\
-\left(T^{-}\right)^{-1} R^{+} & \left(T^{-}\right)^{-1}
\end{array}\right),
$$

Further, $S^{i}$ are the matrices of the phase shifts inside the slab,

$$
S^{i}=\left(\begin{array}{cc}
S^{+} & 0 \\
0 & S^{-}
\end{array}\right), S^{ \pm}=\left\{e^{ \pm i k \tau_{s} v_{S} d_{i}}\right\},
$$

and the matrix $G$ is the transfer matrix of the field (1) through the grating. It can be obtained similarly to [8]. To obtain the vector of reflected Floquet harmonics, we use Eq.(2),

$$
\begin{aligned}
& r=-\left(A^{I V}\right)^{-1} A^{I I I} a^{\text {incident }}, \\
& t=A^{I I} a^{\text {incident }}+A^{I} r .
\end{aligned}
$$


Note that as our mathematical approach is similar to [8] then the resulting matrix equation (7) is the Fredholm second kind equation.

Consider now the eigenvalue problem. In the classical formulation one is required to find the complex-valued natural frequencies as eigenvalues. As discussed in [6], if a pumping is introduced in the wires to compensate for the losses, the imaginary part of the natural frequency can be brought to zero. This corresponds to the threshold of the lasing understood as the emission of non-attenuating in time natural field. In $[5,6]$, we have offered a specific eigenproblem tailored for the lasers as open resonators equipped with active regions.

In particular case considered in this paper, we assume that the wires of embedded grating have refractive index with a strictly negative imaginary part, $v=\alpha-i \gamma$, while the refractive index of host slab stays real, $v_{s}$. Then, after substituting the modified material parameters into the earlier discussed scattering problem and deriving the expressions similar to (1)-(6) but without any incident field, we come the determinantal equation

$$
\operatorname{det}\left[A^{I V}(\sigma, \gamma)\right]=0 \text {. }
$$

Then the lasing frequency and threshold $\left(\sigma_{j}, \gamma_{j}\right)$ are the roots of (9). According to [6], the lasing threshold is inversely proportional to the Q-factor of corresponding natural mode. Therefore we are interested in finding the mode with lowest threshold.

The roots of (9) are found using the Newton method starting from the initial-guess values in such a manner that the frequency is taken from the resonance value in the scattering problem and the threshold is taken as arbitrary small number.

The considered problem has two limiting cases. Firstly if the refractive indices are equal, $v=v_{s}$, then we have a homogeneous dielectric slab $(G=I)$ whose reflectance and transmittance do not depend on the polarization at the normal incidence of plane wave. Secondly, if the slab refractive index is $v_{s}=1$, then $F^{1,2}=I$ and we have a grating in free space. In the latter case, the asymptotic expressions for eigenvalues are known for the $H$-polarization,

$$
\begin{aligned}
& \sigma_{m}^{0}=m-\frac{\pi^{8} m^{8}\left(\alpha^{2}-1\right)^{2}}{2 \xi^{8}}, \\
& \gamma_{m}^{0}=\frac{\pi^{4} m^{3}\left(1-\alpha^{2}\right)^{2}(\pi m-1)}{8 \alpha \xi^{4}}, \\
& \sigma_{m}^{n}=m-\frac{C_{n}^{m}}{\xi^{8 n}}\left(\frac{\alpha^{2}-1}{1+\alpha^{2}}\right)^{2}, \gamma_{m}^{n}=\frac{C_{n}^{m}}{\xi^{4 n}} \frac{\alpha^{2}-1}{1+\alpha^{2}},
\end{aligned}
$$

and for the $E$-polarization,

$$
\begin{aligned}
& \sigma_{m}^{0}=m-\frac{\pi^{4} m^{4}\left(\alpha^{2}-1\right)^{2}}{2 \xi^{4}}, \\
& \gamma_{m}^{0}=\frac{\pi^{2} m\left(1-\alpha^{2}\right)^{2}(\pi m-1)}{8 \alpha \xi^{2}}, \\
& \sigma_{m}^{n}=m-\frac{C_{n}^{m}}{\xi^{4 n}}\left(\frac{\alpha^{2}-1}{1+\alpha^{2}}\right)^{2}, \gamma_{m}^{n}=\frac{C_{n}^{m}}{\xi^{2 n}} \frac{\alpha^{2}-1}{1+\alpha^{2}},
\end{aligned}
$$

where $\xi=p / a$ is the relative separation between cylinders. Formulas (11) and (13) are given for the higher order grating modes with $n \geq 1$. Here the lower index $m \geq 1$ denotes the number of the branch point and the upper one $n \geq 1$ does the mode order. Thus, the lasing threshold is inversely proportional to the value of relative separation that means for sparser gratings we have smaller thresholds or higher Q-factors. Additionally the expressions for the thresholds contain the term $1-v^{2}$ that is the contrast between the refractive indices of the host slab and the grating. The effect of the small contrast will be studied further. Looking at the frequency expressions, one can notice that for the higher orders $n$ they very rapidly approach $m$. As a result, such modes are very difficult for investigation as even for small values of $\xi$ their frequencies merge with the corresponding branch point.

\section{Scattering and eigenvalues}

Firstly we investigated the scattering problem for the geometry shown in Fig. 1. Here we assumed that $p=d$, $d_{1}=d_{2}=1$ and $v=2$. We have varied the slab refractive index $v_{s}$ from 1 to 2 . In other words, the variation has been conducted between two limiting cases: the wire grating in free space $\left(v_{s}=1\right.$ that corresponds to the bottom line of reflectance reliefs in Figs $2 \mathrm{a}$ and $2 \mathrm{~d}$ and the homogeneous slab $\left(v_{s}=2\right.$ that corresponds to the upper lines of the same figures). In the intermediate cases, the interaction of the slab modes and the grating modes takes place. However with increasing the slab refractive index the grating resonances become sharper and they vanish on the reliefs in Fig. 2 just because of finite resolution. The natural frequencies and thresholds

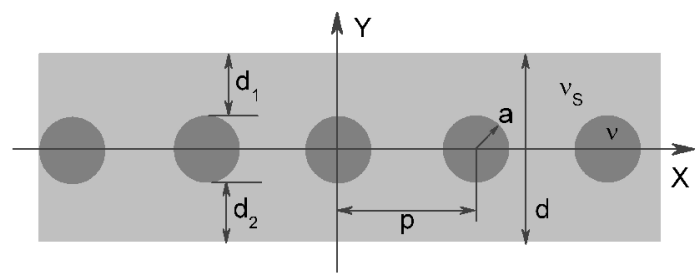

Fig. 1. The cross-sectional geometry of a periodically structured dielectric slab. 
have been calculated for the explained above lasing eigenvalue problem, where the gain was added to the grating wires by adding to their refractive index a negative imaginary part $\gamma$. Note that the lasing frequencies accurately follow the traces of the grating resonances on the reliefs of reflectance; still the lasing threshold shows extremely rapid fall if coming to the case where the real part of active-region refractive index and that of the host slab are equal. Here it should be noted then in the $H$-polarization case the resonances are sharper and the corresponding thresholds lower than in the $E$-case.
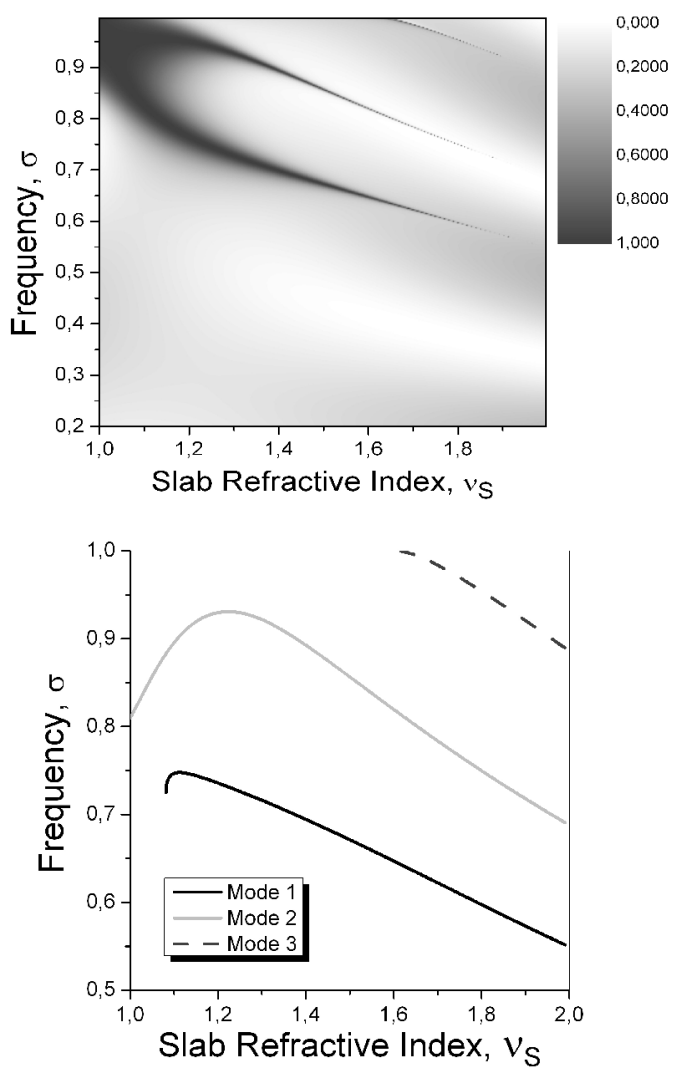

$b$

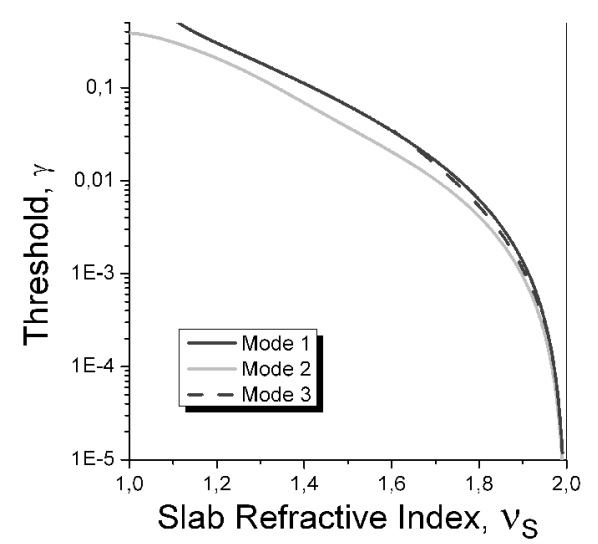

Fig. 2. Characteristics of the dielectric slab with embedded grating having $v=2$ (for reliefs of reflectance (a)(d)) and $v=2-i \gamma$ (for the grating-mode eigenvalues (b), (c) and (e), (f)) and $d_{1}=d_{2}=a$ or $d=p=4 a$. The $E$ - (a),(b),(c) and the $H$-polarization (d), (e),(f).

(C) 2014, V. Lashkaryov Institute of Semiconductor Physics, National Academy of Sciences of Ukraine
We have been also interested in the influence of the slab width on the scattering properties and eigenvalues. In Fig. 3, we investigated the structure consisting of a wire grating with $v=2$ and period $p=4 a$ placed in the middle of a slab with $v_{s}=1.6$ and variable width. Reliefs of reflectance presented in Figs. 3a and 3d show that widening the slab excites extremely high-Q grating modes of higher orders (11), (13). The background is formed by the broader ridges representing the slab modes, which do not depend on the polarization and thus look similar for the both reliefs. In the case of $\mathrm{H}$ polarization the ridges of different nature experience
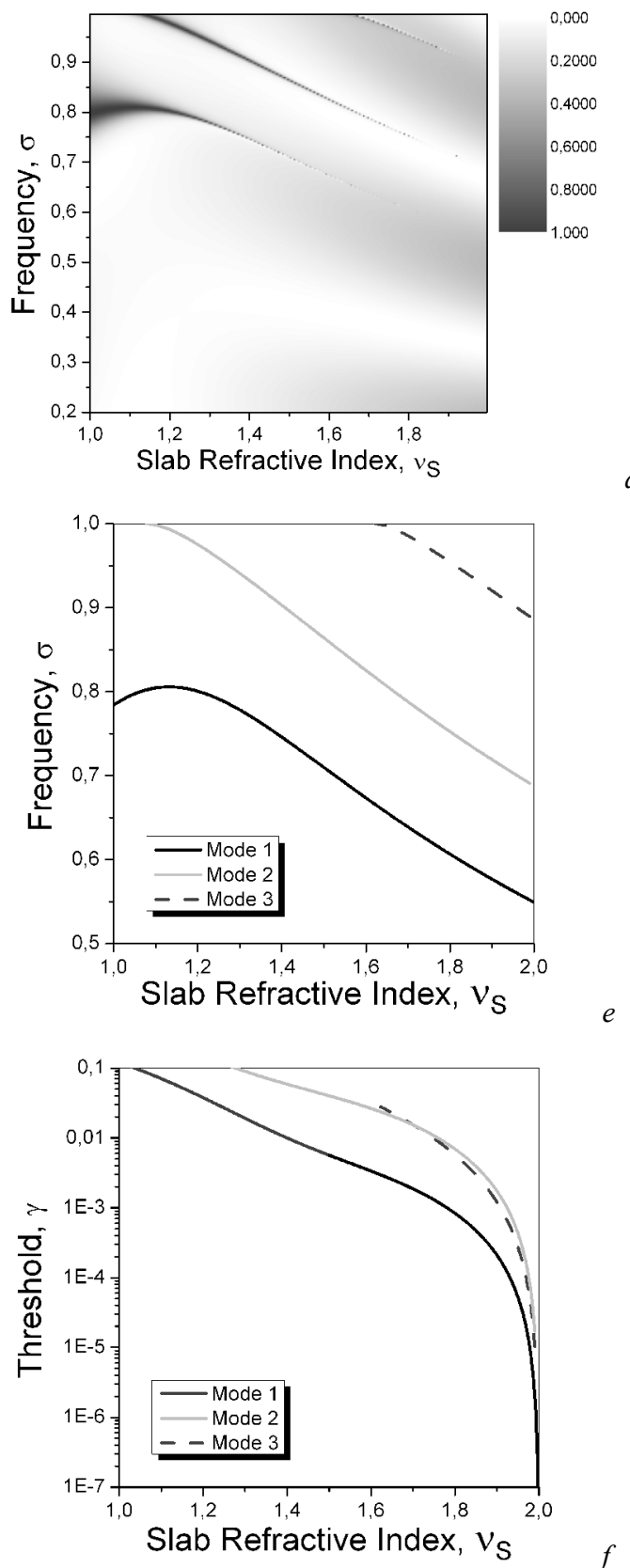

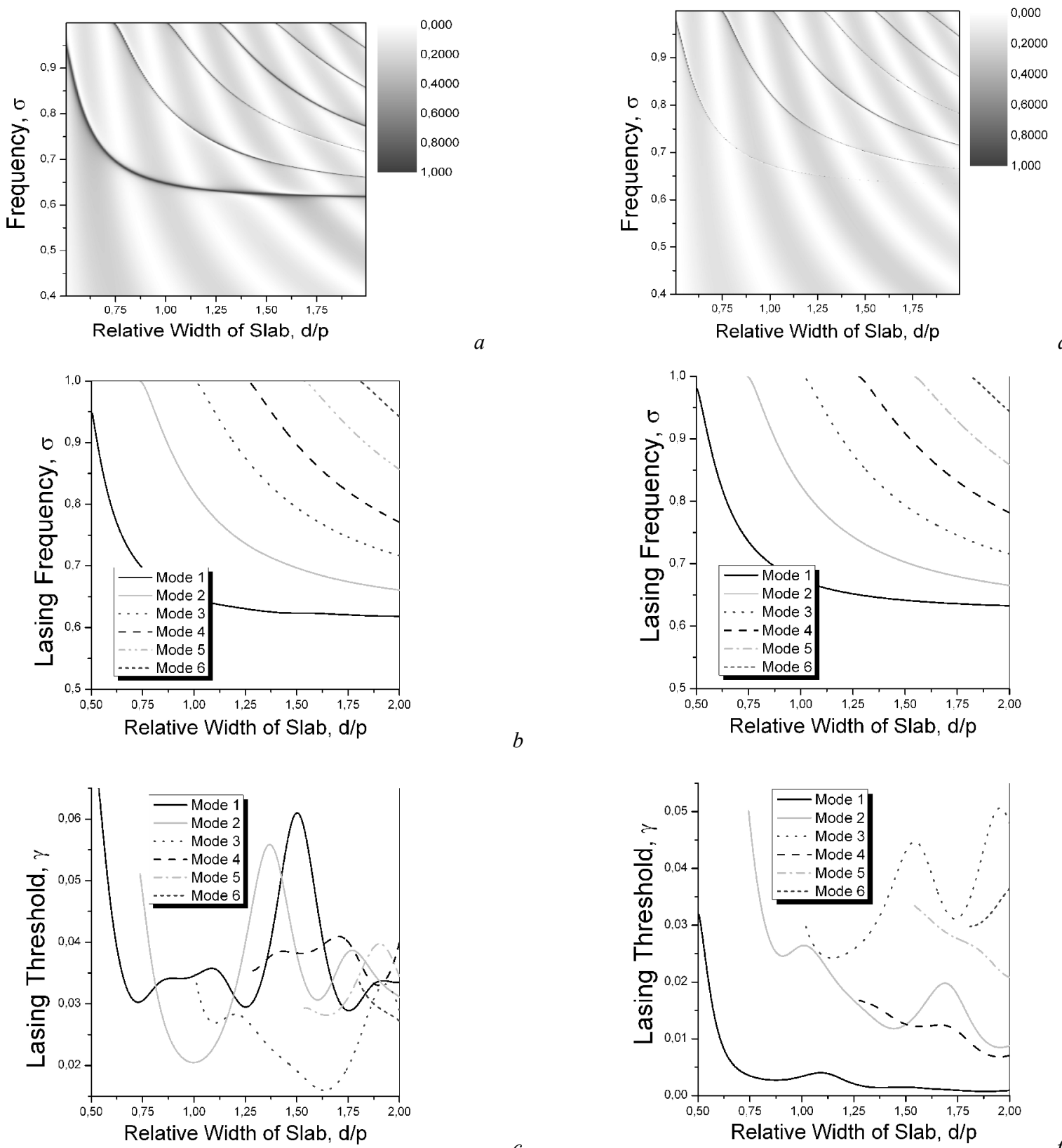

Fig. 3. Characteristics of the dielectric slab of refractive index $v_{\mathrm{s}}=1.6$ with embedded grating having $v=2$ (for reliefs of reflectance (a)(d)) and $v=2-i \gamma$ (for the lasing frequencies (b),(e) and thresholds (c),(f)). Period is $p=4 a$. The $E$-polarization (a),(b),(c) and the $H$-polarization (d),(e),(f).

weaker coupling than in the $E$-case. The grating modes have been investigated using the eigenvalue problems see Figs. 3b, 3c, 3e, and 3f. Here again the lasing frequencies almost repeat the behavior of resonant ridges in the associated scattering problem. The lasing thresholds show more complicated behavior. Mostly they go up as the slab shrinks. However they drop if they approach the slab-mode eigenvalues [9] (this corresponds to the intersection of the areas of high reflectivity of the slab). In this case the filed inside of the slab rises, and then the grating-shaped active region occurs in the $E$-field maximum thus the overlap is better. This entails smaller threshold values as we have shown in [6].

\section{Conclusion}

We have studied numerically the scattering of the $E$ - and $H$-polarized plane waves incident normally at a dielectric slab with an embedded grating of subwavelength circular cylinders of different dielectric material. The reflectance of these composite periodic

\section{(C) 2014, V. Lashkaryov Institute of Semiconductor Physics, National Academy of Sciences of Ukraine}


scatterers demonstrates a remarkable interplay of the slab and grating resonances. We have also studied the natural modes responsible for these resonances. They have been computed as eigenvalues of the lasing problem, where the wire material is assumed to have gain characterised with the aid of the "active" imaginary part of the refractive index. We have shown that varying the host slab width one can excite higher-order grating resonances that are less accessible if the grating is located in free space. Interaction of the grating modes with the slab modes is able to produce sharper resonances of reflectance due to increased modal Qfactors and correspondingly able to lower the thresholds of their lasing.

\section{Acknowledgement}

This work has been partially supported by the National Academy of Science of Ukraine via the State Target Program "Nanotechnologies and Nanomaterials".

\section{References}

1. V. Twersky, On scattering of waves by the infinite grating of circular cylinders // IEEE Trans. Antennas Propagation, 10(3), p. 737-765 (1962).

2. O. Kavaklioglu, On diffraction of waves by the infinite grating of circular dielectric cylindersat oblique incidence: Floquet representation // J. Modern Phys. 48(1), p. 125-142 (2001).

3. K. Yasumoto, H. Toyama, T. Kushta, Accurate analysis of 2-D electromagnetic scattering from multilayered periodic arrays of circular cylinders using lattice sums technique // IEEE Trans. Antennas Propagation, 52(10), p. 2603-2611 (2004).

4. R. Gomez-Medina, M. Laroche, J.J. Saenz, Extraordinary optical reflection from sub- wavelength cylinder arrays // Opt. Exp. 14(9), p. 3730-3737 (2006).

5. V.O. Byelobrov, J. Ctyroky, T.M. Benson, A. Altintas, R. Sauleau, A.I. Nosich, Low-threshold lasing modes of an infinite periodic chain of quantum wires // Opt. Lett. 35(21), p. 3634-3636 (2010).

6. E.I. Smotrova, V.O. Byelobrov, T.M. Benson, P. Sewell, J. Ctyroky, A.I. Nosich, Optical theorem helps understand thresholds of lasing in microcavities with active regions // IEEE J. Quantum Electronics, 47(1), p. 20-30 (2011).

7. V.O. Byelobrov, T.M. Benson, A.I. Nosich, Near and far fields of high-quality resonances of an infinite grating of sub-wavelength wires // Proc. European Conf. Microwaves (EuMC-11), Manchester, 2011, p. 858-861.

8. D.M. Natarov, V.O. Byelobrov, R. Sauleau, T.M. Benson, A.I. Nosich, Periodicity-induced effects in the scattering and absorption of lilght by infinte and finite grating of circular silver nanowires // Opt. Exp. 19(22), p. $22176-22190$ (2011).

9. V.O. Byelobrov, A.I. Nosich, Mathematical analysis of the lasing eigenvalue problem for the optical modes in a layered dielectric cavity with a quantum well and distributed Bragg reflectors // Optical and Quantum Electronics, 39(10-11), p. 927-937 (2007).

10. K. Yasumoto, T. Kushta, H. Toyama, Reflection and transmition from periodic composite structure of circular cylinders // Proc. Intern. Symp. Physics Eng. Microwaves, MM and Sub-MM Waves (MSMW'2001), Kharkiv, p. 96-101, 2001.

11. A.I. Nosich, Radiation conditions, limiting absorption principle, and general relations in open waveguide scattering // J. Electromagn. Waves Applications, 8(3), p. 329-353 (1994). 